\title{
Verbesserte Magnetresonanz-Myelografie durch Bildfusion
}

\author{
Improved Magnetic Resonance Myelography Using Image Fusion
}

Autoren

Institute
K. Eberhardt ${ }^{1}$, O. Ganslandt ${ }^{2}$, A. Stadlbauer ${ }^{2,3}$

MRT-Kompetenzzentrum, Krankenhaus Schloss Werneck

2 Neurochirurgische Klinik, Universität Erlangen-Nürnberg, Erlangen

3 Zentrales Institut für Radiologie, Diagnostik und Interventionelle Therapie, Landesklinikum St. Pölten, St. Pölten, Österreich

\author{
Key words \\ - spine \\ - myelography \\ - MR imagingimage fusion \\ post-myelography CT
}

eingereicht 30.8 .2012

akzeptiert 19.11.2012

\section{Bibliografie}

DOI http://dx.doi.org/ 10.1055/s-0032-1330440

Online-Publikation: 24.1.2013

Fortschr Röntgenstr 2012; 184:

333-339 @ Georg Thieme

Verlag KG Stuttgart · New York . ISSN 1438-9029

\section{Korrespondenzadresse \\ Dr. Knut Eberhardt}

MRT-Kompetenzzentrum, Krankenhaus Schloss Werneck Balthasar-Neumann-Platz 1 97440 Werneck

Tel.: ++ 49/97 22/947324

Fax: ++49/97 22/947326

\section{Zusammenfassung \\ $\nabla$}

Ziel: Ziel war es zu zeigen, dass der bei der MRMyelografie durch die starke T2-Gewichtung auftretende Nachteil der fehlenden anatomischen Zuordnung, durch eine Bildfusion sowie durch in koronarer Phasenkodierrichtung generierte Quellbilder behoben werden kann, wodurch eine dem Goldstandard - dem postmyelografischen CT - vergleichbare Bildinformation erzielt werden kann.

Material und Methoden: Untersucht wurden insgesamt 110 Patienten mit extraduralen Pathologien der HWS und der LWS. Alle Patienten erhielten eine 3D-MR-Myelografie und ein postmyelografisches CT. Anschließend wurden die MRT-Datensätze durch Bildfusion und Rekonstruktionen nachverarbeitet und mit den entsprechenden Schnittbildern des postmyelografischen $C T$ verglichen.

Ergebnisse: Die in der Arbeit verwendete Visualisierungstechnik (3D-MR-Myelografie) ist hierbei in der Lage intradurale Substrukturen hochauflösend und artefaktfrei darzustellen. Die erreichbaren Visualisierungsergebnisse sind mit dem aktuellen Goldstandard (postmyelografisches CT) vergleichbar. Eine anatomische Zuordnung ist durch Verwendung überlagerter MRT-Sequenzen im Rahmen einer Bildfusion möglich. Die hierzu notwendigen Arbeitsschritte lassen sich rasch umsetzen und sind auf kommerziellen Workstations verfügbar.

Schlussfolgerung: Durch die Fusion von unterschiedlichen MRT-Sequenzen entstehen neue Qualitäten bei der Visualisierung komplexer 3D-Datensätze. Insbesondere können der konventionellen Myelografie einschließlich der postmyelografischen $\mathrm{CT}$ vergleichbare Visualisierungsergebnisse in Form der sogenannten 3D-MR-Myelografie realisiert werden. Der durch die starke T2-Gewichtung verlorene Bezug zu anatomischen Leitstrukturen kann durch Fusion mit einem weiteren MRT-Datensatz ausgeglichen werden.

\section{Abstract \\ $\nabla$}

Purpose: To demonstrate that the disadvantage of missing anatomical information in heavily T2weighted MR myelography images can be eliminated by image fusion and phase encoding in the coronal direction of the source images, resulting in MR myelography images comparable to the gold standard, i. e., post-myelography CT.

Materials and Methods: This study included 110 patients suffering from extradural pathologies of the cervical and lumbar spine. All patients were investigated using 3D MR myelography and postmyelography CT. The MRI data were post-processed using image fusion and reconstruction algorithms and were compared to the corresponding images of post-myelography CT.

Results: Our approach for visualization (3D MR myelography) was able to depict intradural structures in high spatial resolution and without artifacts. The results of our visualization approach were comparable to the gold standard - postmyelography CT. Anatomical correlation was reached by image fusion of different MR data sets. The required post-processing steps were performed quickly and were available on a commercial workstation.

Conclusion: Image fusion of different MR data sets allows for visualization of 3D data sets with enhanced quality. The results for the visualization of MR myelography in particular are comparable to conventional myelography and post-myelography CT. The missing anatomical information in heavily T2-weighted MR myelography images can be compensated by image fusion with conventional MRI. 


\section{Einleitung \\ $\nabla$}

Neuronale Kompressionssyndrome spielen eine große Rolle in der Pathophysiologie verschiedenster Krankheiten im Bereich der Wirbelsäule. Die Klinik von Schmerzsyndromen ist oft uncharakteristisch und fokalneurologisch spezifischen Dermatomen unzureichend zuzuordnen. Trotz erheblich verbesserter Diagnostik bestehen bei Wirbelsäulenerkrankungen noch große Diskrepanzen zwischen Klinik und Bildgebung. So haben Patienten mit großen Raumforderungen oft nur geringe klinische Symptome und umgekehrt Patienten ohne relevanten morphologischen Befund in der Bildgebung starke klinische Beschwerden $[1,2]$. Auch eine experimentelle Tierstudie kommt zu diesem Ergebnis [3]. Von einer verbesserten Schnittbilddiagnostik muss deshalb erwartet werden, dass Klinik und morphologischer Befund in Zukunft besser in Übereinstimmung gebracht werden. In der Vergangenheit galt zunächst die lumbale Myelografie als Goldstandard. Bei diesem invasiven Verfahren ist die diagnostische Sicherheit allerdings eingeschränkt $[4,5]$ und die Gefahr von allergischen Reaktionen auf das Kontrastmittel muss beachtet werden [6]. Durch die Einführung und Weiterentwicklung [7] der Computertomografie ergab sich die Möglichkeit, eine konventionelle Myelografie mit einer digitalen Schnittbilddiagnostik zu kombinieren (postmyelografisches CT, pm-CT). Vergleichende Studien von Rainko et al. [8] und Kampmann et al. [9] zeigen schon früh die Überlegenheit dieser Technik.

Die Kernspintomografie bietet hingegen die beste Weichteilauflösung und wurde deshalb zunächst zur Diagnose intraspinaler Raumforderungen, insbesondere neuronaler Kompressionssyndrome und Tumoren eingesetzt $[10,11]$. Ein großer Vorteil gegenüber den anderen Techniken ist die fehlende Strahlenexposition des Patienten [12-14]. Durch die Entwicklung stark T2gewichteter Sequenzen war es möglich, den Liquorraum mit ausreichender Auflösung dreidimensional und frei von überlagerten Pulsationsartefakten darzustellen. Die MR-Myelografie visualisiert ein der konventionellen Myelografie vergleichbares Bild [15]. Da es sich um isotrope 3D-Datensätze handelt, können problemlos verschiedene multiplanare Rekonstruktionen erstellt werden. Nachteil dieser Sequenzen ist der Verlust der Umgebungsinformation, der eine anatomische Zuordnung unmöglich macht, zumal die bevorzugte Phasenkodierrichtung der Quellbilder koronar ist, um eine optimierte Bildqualität zu erhalten. In dieser Arbeit sollte deshalb geprüft werden, ob eine automatisierte, schwellenwertbasierte Nachverarbeitung im Rahmen der sogenannten MR-Myelografie möglich ist und ob durch Bildfusion eine ausreichende anatomische Zuordnung erreicht werden kann, sodass sekundäre, axial und parallel zum Zwischenwirbelraum ausgerichtete, den postmyelografischen Bildern vergleichbare MRT-Rekonstruktionsbilder erzeugt werden können. Hierzu wurden Patienten mit verschiedenen extraduralen Pathologien im Bereich der Wirbelsäule mittels pm-CT und MR-Myelografie vergleichend untersucht.

\section{Material und Methode \\ $\nabla$}

\section{Patienten}

Im Rahmen der Studie wurden 110 Patienten mit verschiedenen extraduralen Pathologien untersucht. 60 Patienten (21 Frauen und 39 Männer, Durchschnittsalter 60,2 $\pm 12,1$ Jahre) erhielten Untersuchungen der HWS und 50 Patienten (15 Frauen und 35 Männer, Durchschnittsalter 61,6 $\pm 11,9$ Jahre) der LWS. Alle
Patienten gaben nach einer Aufklärung ihr schriftliches Einverständnis zur Teilnahme an der Studie.

\section{Myelografie und postmyelografisches CT}

Die konventionale Myelografie wurde in Bauchlage durchgeführt. Das nicht ionische Kontrastmittel (10 ml Isovist 300, Schering AG, Berlin, Deutschland) wurde intrathekal verabreicht. Zur Darstellung der Lendenwirbelsäule wurden 4 Standardprojektionen in anterior-posteriorer, lateraler und LAO oder RAO Orientierung gemacht. In allen Patienten wurde anschließend ein postmyelografisches CT angefertigt.

\section{MR-Myelografie}

Die MR-Untersuchungen wurden an einem 1,5 Tesla-Ganzkörper-MR-Scanner (Sonata, Siemens, Erlangen, Deutschland) in Kombination mit der aus 6 Spulenelementen bestehenden CPSpine-Array-Spule durchgeführt. Um intradurale Strukturen (Nerven, Gefäße) vom umgebenden Liquorraum differenzieren zu können, wurde eine stark T2-gewichtete Sequenz verwendet, wobei dann die Strukturen als Aussparung im signalreichen Liquorraum erscheinen. Zu diesem Zweck wurde eine 3D-HalfFourier-Aquisition-Single-Shot(HASTE)-Sequenz durchgeführt. Folgende Sequenzparameter wurden für die Aufnahmen verwendet: $T R=10000 \mathrm{~ms}, \mathrm{TE}=165 \mathrm{~ms}$, Schichtdicke $=0,5 \mathrm{~mm}$, Flip Winkel von $180^{\circ}$, Voxelgröße von $0,5 \times 0,5 \times 0,5 \mathrm{~mm}^{3}$ bei einer $256 \times 256$ Matrix und einem Field of View (FoV) von $120 \times 120 \mathrm{~mm}^{2}$. Die Orientierung des Volume of Interest (VoI) war in koronarer Schichtebene (Phasenkodierrichtung). Die Messzeit betrug $10 \mathrm{~min}$. Die Sequenz mit einer frequenzselektiven Fettunterdrückung und einem koronaren und axialen Sättigungspuls (in der HWS) bzw. koronaren Sättigungspuls (in der LWS) versehen. Auf diese Weise konnten die im Bereich der HWS üblicherweise auftretenden Schluck- und Gefäßpulsationsartefakte (supraaortale Gefäße) sowie im Bereich der LWS auftretende Atmungsartefakte zuverlässig unterdrückt werden.

Als anatomische Basisdatensätze wurden einerseits eine Standard-Spin-Echo-Sequenz mit T2-Gewichtung in sagittaler Orientierung und folgenden Parametern: TR $=6400 \mathrm{~ms}$, TE $=165 \mathrm{~ms}$, Schichtdicke $=3 \mathrm{~mm}$, FoV $=250 \times 250 \mathrm{~mm}^{2}$, Matrix $=256 \times 256$, bei einer Messzeit von 1:30 min sowie mit T1-Gewichtung und folgenden Parametern verwendet: TR $=500 \mathrm{~ms}, \mathrm{TE}=27 \mathrm{~ms}$, Schichtdicke $=3 \mathrm{~mm}$, FoV $=250 \times 250 \mathrm{~mm}^{2}$, Matrix $=256 \times 256$, bei einer Messzeit von $56 \mathrm{~s}$. Außerdem wurde zusätzlich eine 3D-MEDIC (Multi Echo Data Imaging Combinaton)-Sequenz durchgeführt mit folgenden Parametern: TR=81 ms; TE $=27 \mathrm{~ms} ; \alpha=14^{\circ}$; Schichtdicke $=3 \mathrm{~mm}$; FoV $=200 \times 200 \mathrm{~mm}^{2}$; Matrix $=256 \times 256$ bei einer Messzeit von ca. 3:30 min.

\section{Nachverarbeitung der MR-Daten mittels Bildfusion}

Um eine exakte anatomische Zuordnung zu erreichen, wurde den 3D-HASTE-Datensätzen jeweils ein Datensatz einer entsprechenden sagittalen T2-gewichteten TSE-Sequenz und/oder 3D-MEDIC-Sequenz überlagert und fusioniert. Die Bilddatenfusion erfolgte an einer kommerziellen Workstation (Leonardo, Siemens, Erlangen, Deutschland). Hierfür wurde die in der Workstation integrierte Funktion „3D Fusion“ verwendet.

Die „3D Fusion“ beinhaltet standardmäßig eine automatische Überlagerungskorrektur. Diese arbeitet nach folgendem Algorithmus: Um 2 Volumendatensätze automatisch zu registrieren, wird eine Metrik zur Erfassung der stochastischen Abhängigkeit zwischen 2 Volumina verwendet, genannt „Mutual Information“. Diese Metrik stammt ursprünglich aus der Signaltheorie. Bei 
dem automatischen Algorithmus nutzt man aus, dass sich die korrekte Registrierung aus mathematischer Sicht dadurch erkennen lässt, dass hier die stochastische Abhängigkeit zwischen den beiden Datensätzen ein Extremum annimmt. Es wird eine Lage in beiden Volumina gesucht, in der die zugrunde liegende Metrik eine maximale geometrische Übereinstimmung annimmt. Ein Problem, das sich ausreichend gut mathematisch lösen lässt. Um zu verhindern, dass die Größe des gemeinsamen Schnittvolumens bei vergleichbarer Gewichtung einen nennenswerten Einfluss auf das Registrierungsergebnis hat, wird eine Variante gewählt, der eine geometrische Mustererkennung zugrunde liegt („Normalized Mutual Information“). Alternativ besteht die Möglichkeit bei Daten, bei denen die automatische Überlagerung nicht ausreichend ist, mit einer zweiten Funktion eine manuelle Überlagerungskorrektur durchzuführen. Die Funktionalität besteht darin, dass jeweils der überlagerte Datensatz anhand 8 vorgegebener geometrischer Bildachsen verschoben werden kann. Die anatomische Zuordnung kann jederzeit über die abgebildeten 3 Ebenen kontrolliert werden. Weiterhin besteht im Rahmen der Fusionsfunktionalität die Möglichkeit im fusionierten Datensatz die jeweiligen Grunddatensätze (MPR von 3D-HASTE und TSE) beliebig farbig zu kodieren und gewichtet darzustellen (T2-gewichtet bei 3D-HASTE und T2-TSE, bzw. T1-gewichtet bei T1-TSE).

Nach der Bildfusion wurden die folgenden Arbeitsschritte durchgeführt: 1.) Festgelegen des Zwischenwirbelraums, der aufgrund der Datenfusion eindeutig definiert werden konnte; 2.) Festlegen des Zentrums des Zwischenwirbelraums als Mittelschicht, wobei danach von dieser Position jeweils zur Mittelschicht ergänzend 4 Schichten nach kranial und 4 nach kaudal im Abstand von $2 \mathrm{~mm}$ mittels MPR (multiplanare Rekonstruktion) erstellt wurden. Die Schichtdicke betrug jeweils $3 \mathrm{~mm}$; und 3.) Überlagern eines fusionierten Bildes mit den axialen Schichtebenen, um die anatomische Zuordnung der jeweiligen axialen MPR zu dokumentieren ( $\bullet$ Abb. 1).

\section{Evaluierung und Auswertung}

Die Beurteilung der Abgrenzbarkeit intraduraler Strukturen sowie der Darstellbarkeit extraduraler Pathologien sowie die Bewertung der angewendeten Strategie zur Nachverarbeitung der MR-Bilddaten erfolgte interdisziplinär durch einen erfahrenen Neuroradiologen, einen erfahrenen Neurochirurgen und einen erfahrenen Medizinphysiker mit Schwerpunkt MR-Bildgebung im Konsensus. Hierfür wurden die mittels MPR axial reformatierten 3D-HASTE-Schnittbilder mit axialen Schnittbildern des entsprechenden postmyelografischen $\mathrm{CT}$ verglichen. Es galten folgende Bewertungskriterien:

1. Beim Normalbefund erfolgte die Bewertung anhand der Lage und der Abgrenzbarkeit intraduraler Substrukturen (Nervenwurzelfilamente, Nervenwurzelstränge) und
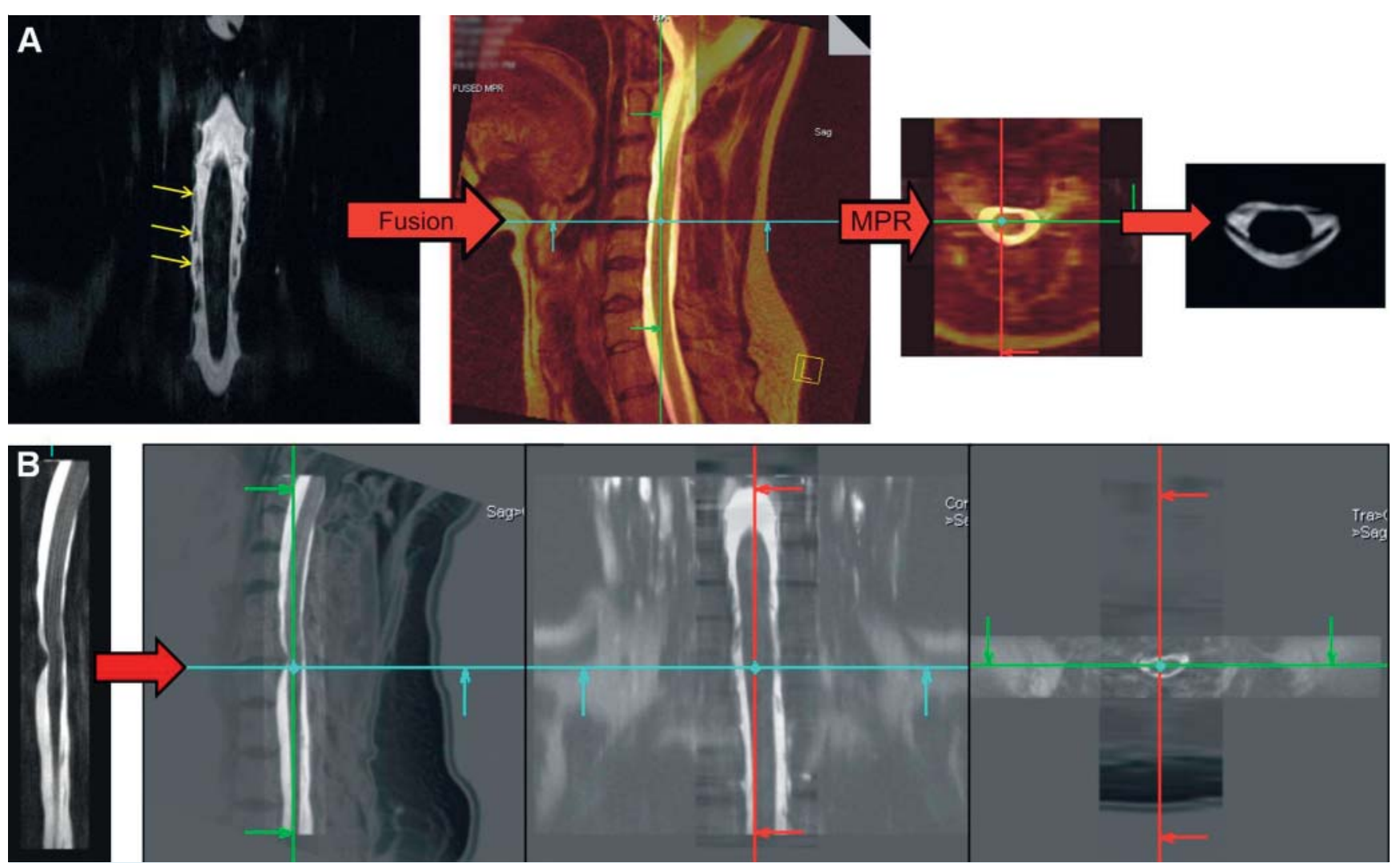

Abb. 1 Nachverarbeitung der MR-Daten. a Von links nach rechts: Liquorbild der 3D-HASTE-Sequenz eines Patienten im Bereich der HWS. Im Bereich des Liquors sind deutlich die Aussparungen der Spinalnervenwurzeln zu erkennen (gelbe Pfeile). Gut erkennbar ist auch der Nachteil der 3D-HASTESequenz: die fehlende anatomische Zuordnung. Durch Fusion entsteht eine Überlagerung mit dem sagittalen T2-gewichteten TSE-Datensatz. Nach MPR erhält man axiale Ergebnisbilder mit noch erkennbarer anatomischer Überlagerung bzw. (ganz rechts) wahlweise auch überlagerungsfreie
HASTE-betonte Bilder mit hoher Detailauflösung. b Links: Sagittale Darstellung des 3D-HASTE-Datensatzes eines Patienten mit lateralem Bandscheibenvorfall. Auch hier wieder gut zu erkennen, dass die anatomische Zuordnung fehlt. Rechts: Fusion der 3D-HASTE-Aufnahme mit einem TSEDatensatz, um die anatomische Zuordnung wiederherzustellen. Der Bezug zu den knöchernen Strukturen ist wieder eindeutig (sagittale, koronare und axiale Schnittebene). 
2. bei Pathologien erfolgte die Bewertung anhand der Abgrenzbarkeit der intraduralen Substrukturen und des Kompressionsausmaßes oder der Verlagerung der intraduralen Strukturen.

Folgende Fragen sollten beantwortet werden:

a) Lassen sich intradural gelegene Strukturen (Nervenfasern, Gefäße) in allen 3 Rekonstruktionsebenen der MPR ausreichend abgrenzen?

b) Ist im Rahmen der entwickelten Nachverarbeitungsstrategie eine automatische Fusion ausreichend oder ist eine manuelle Korrektur notwendig (wobei als exakte Fusion eine solche betrachtet wurde, bei der die fusionierten Sequenzen im Wirbelkanal sich passgenau in allen drei Ebenen anatomisch überlagerten)?

c) Sind die axialen MPR der 3D-HASTE-Sequenz mit dem postmyelografischen CT bezüglich der Abgrenzung der intraduralen Strukturen vergleichbar und damit in der Aussagekraft hinreichend?

\section{Ergebnisse}

$\nabla$

Bei den 60 Patienten mit Pathologien im Bereich der HWS wurden insgesamt 360 Segmenthöhen (6 Segmente pro Patient) untersucht. Bei 151 Segmenten (41,9\%) ergab sich ein pathologischer Befund wobei sich im Detail folgende Pathologien zeigten: 53 (14,7\%) mediale Bandscheibenvorfälle, 36 (10\%) laterale Bandscheibenvorfälle 24 (6,7\%) Bandscheibenprotrusionen und 38 (10,6\%) sonstige Diagnosen (Veränderungen im Bereich der Facettengelenke oder der Ligamente, Raumforderungen). Im Bereich der LWS ergab sich in den 50 Patienten bei 250 Segmenthöhen (5 Segmente pro Patient) bei 84 Segmenten (33,6\%) ein pathologischer Befund. Im Detail wurden folgende Pathologien gefunden: 12 (4,8\%) mediale Bandscheibenvorfälle, 26 (10,4\%) laterale Bandscheibenvorfälle, 25 (10\%) Bandscheibenprotrusionen und $21(8,4 \%)$ sonstige Diagnosen.

Durch die starke T2-Gewichtung und die damit verbundene hohe Signalstärke des Liquors der 3D-HASTE waren im Bereich der HWS sowohl die vorderen als auch die hinteren Nervenwurzeln der untersuchten Segmente deutlich als signalarme Strukturen abzugrenzen. Bei der LWS war dies in allen Fällen für die Nervenstränge der Cauda equina möglich. Die abgrenzende Darstellung galt als erfolgreich, wenn jedem untersuchten Segment eindeutig eine entsprechende Nervenwurzel zugeordnet werden konnte. Die Möglichkeit, bei einem 3D-Datensatz durch MPR alle Ebenen ohne Verlust von Auflösung als visuelle Kontrolle zur Verfügung zu haben, erwies sich außerdem als großer Vorteil, um Substrukturen sicher zuordnen zu können (vor allem Gefäße betreffend). Für eine automatisierte Form der Fusion von 2 Datensätzen ergab sich, dass bestimmte Voraussetzungen erfüllt sein müssen. Sofern gleiche Gewichtung der Bilder vorliegt, ist eine automatische Fusion problemlos möglich. Liegen die Sequenzen in unterschiedlicher Gewichtung vor, sind bei der Fusion von 2D- mit 3DDatensätzen 2 Voraussetzungen zwingend nötig: gleiches FoV und vergleichbarer geometrischer Referenzpunkt. Bei zwei 3DDatensätzen hingegen muss nur ein vergleichbares FoV vorliegen. Unter Beachtung dieser Voraussetzungen und der verwendeten Parameter ließ sich bei allen Patienten problemlos eine automatische Fusion der Datensätze erstellen, die anhand der MPR in allen 3 Ebenen kontrollierbar waren ( $\bullet$ Abb. 1). Im Anschluss an die Fusion war es bei allen untersuchten Segmenthöhen (360 im Bereich der HWS, 250 im Bereich der LWS) möglich, axiale
MPR Bilder der 3D-HASTE in einer definierten Höhe zu erstellen und zusammen mit einem die Anatomie wiedergebenden Bild abzuspeichern.

- Abb. 2 zeigt ein weiteres Beispiel der Fusion bei einer Patientin mit multiplen Neurinomen. Im 3D-MEDIC-Datensatz wurden zwar die Nervenstränge im Spinalkanal gut dargestellt, er hatte aber eine zu geringe Auflösung für die Darstellung kleiner intraduraler Strukturen. Daher wurde der 3D-MEDIC-Datensatz mit einem 3D-HASTE-Datensatz überlagert. Durch die starke T2-Gewichtung war es so möglich, nicht nur kleine intradurale Strukturen zu erkennen, sondern auch den Verlauf der Nervenstränge zu visualisieren.

Die sekundär mittels MPR axial rekonstruierten Schnittbilder der koronaren Quellbilder wurden mit dem Goldstandard, dem postmyelografischen $\mathrm{CT}$, für Normalbefund und pathologischer Befund verglichen. Ohne pathologischen Befund wurden zwischen postmyelografischem CT und den MPR der 3D-HASTE-Sequenz im Bereich der HWS 1881 Schnittbilder (209 Segmenthöhen) und in der LWS 1494 Schnittbilder (166 Segmenthöhen) verglichen. Es zeigte sich, dass beide Methoden bei der Darstellung von intraduralen Strukturen (Nervenwurzeln) gleiche Ergebnisse lieferten. $\odot$ Abb. 3 demonstriert das Visualisierungsergebnis anhand eines Beispiels.

Bei den Segmenthöhen mit pathologischem Befund wurden 1305 Schnittbilder (151 Segmenthöhen) aus dem Bereich der HWS und 756 Schnittbilder (84 Segmenthöhen) in der LWS zwischen postmyelografischem CT und 3D-HASTE verglichen. Als Kriterien galten die Abgrenzbarkeit und das Kompressionsausmaß oder die Verlagerung der intraduralen Strukturen. Beide Techniken lieferten bei den verschiedenen Pathologien äquivalente Ergebnisse ( $\bullet$ Abb.4). Bei einem HWS-Patienten kam es aufgrund einer hochgradigen Stenose zu einem Kontrastmittelabbruch im postmyelografischen CT, sodass die darunter liegenden Segmente nicht beurteilt werden konnten. Dieser Patient wurde deshalb aus der Auswertung exkludiert.

\section{Diskussion \\ $\nabla$}

In der Literatur findet sich eine Vielzahl von Vergleichen zwischen konventioneller Myelografie, postmyelografischem CT und MR-Myelografie. Kuroki et al. [16] untersuchten eine Gruppe von 40 Patienten mit lumbalem Nervenwurzelkompressionssyndrom. Als Ergebnis zeigte sich, dass sowohl die Darstellung der Strukturen als auch die Bildqualität vergleichbar sind. In Studien von Eberhardt et al. $[17,18]$ konnte an einem Kollektiv von 80 Patienten sogar eine Verbesserung der Darstellung gegenüber der konventionellen Myelografie einschließlich dem postmyelografischem CT gezeigt werden. Untersuchungen von Ferrer et al. [19] bestätigten an einer umfangreichen Studie von 275 Patienten die diagnostische Wertigkeit. So konnte bei 16,7\% die Diagnostik entscheidend verbessert werden. Weitere Arbeiten von Bartlett et al. [20], Birchall et al. [21], Melhem et al. [22] und Wang et al. [23] bekräftigten dieses Ergebnis.

Nachteil bisheriger Methoden ist die mangelnde Darstellung intraduraler Substrukturen. Um auch kleinste intradurale Raumforderungen (z.B. Neurinome) sicher erfassen zu können, ist höchste räumliche Auflösung der anatomischen Substrukturen (Nerven und Gefäße) bei ausreichendem Signal erforderlich [24, 25]. Eine gute Signalabgrenzung der Strukturen ist außerdem essenziell für eine automatisierte Weiterverarbeitung der Datensätze. Eine besondere Bedeutung kommt somit der Wahl der richtigen MR-Se- 

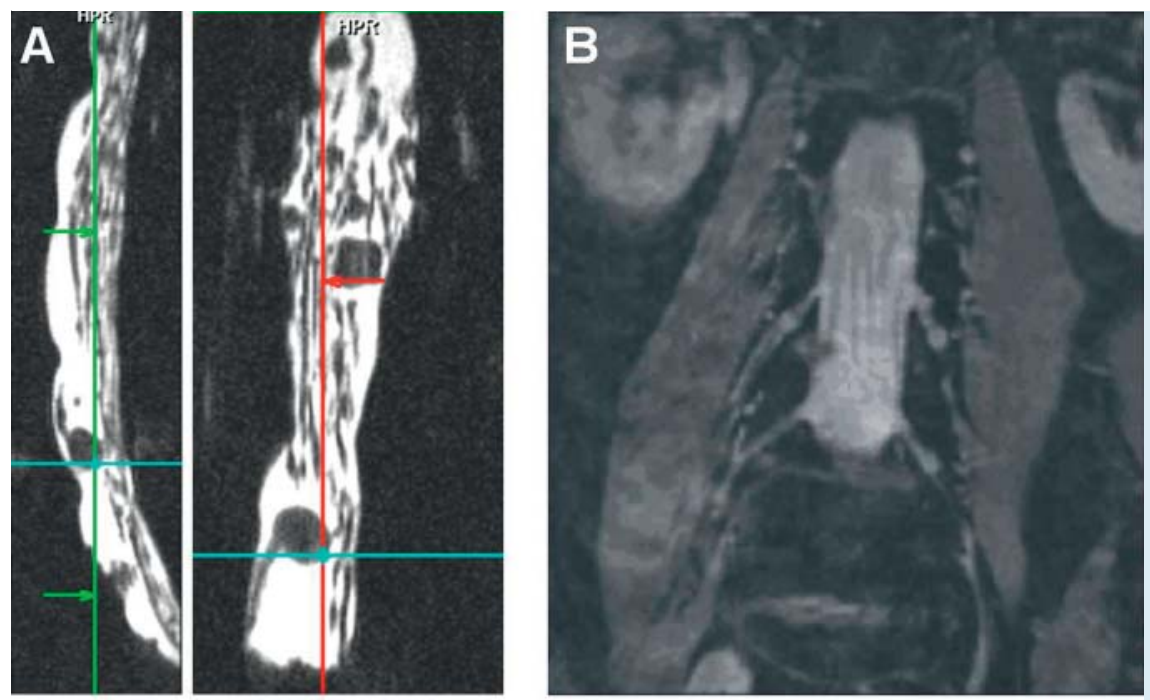

Abb. 2 Nachverarbeitung der MR-Daten. a 3DHASTE-Datensatz einer Patientin mit multiplen Neurinomen. Der Datensatz liefert eine gute Darstellung kleiner intraduraler Strukturen. b 3D-MEDIC-Datensatz derselben Patientin. Hervorzuheben ist die gute Darstellung der aus dem Spinalkanal kommenden Nervenstränge. Nachteil der 3D-MEDIC: Kleine intradurale Strukturen können aufgrund der geringeren Auflösung nicht nachgewiesen werden. c Durch die Fusion des 3D-HASTE- und des 3DMEDIC-Datensatzes erhält man ein vollständiges Bild vom Verlauf der Nervenwurzeln innerhalb des Wirbelkanals sowie nach ihrem Austritt. Eine genaue anatomische Zuordnung ist so intra- und extraspinal möglich.
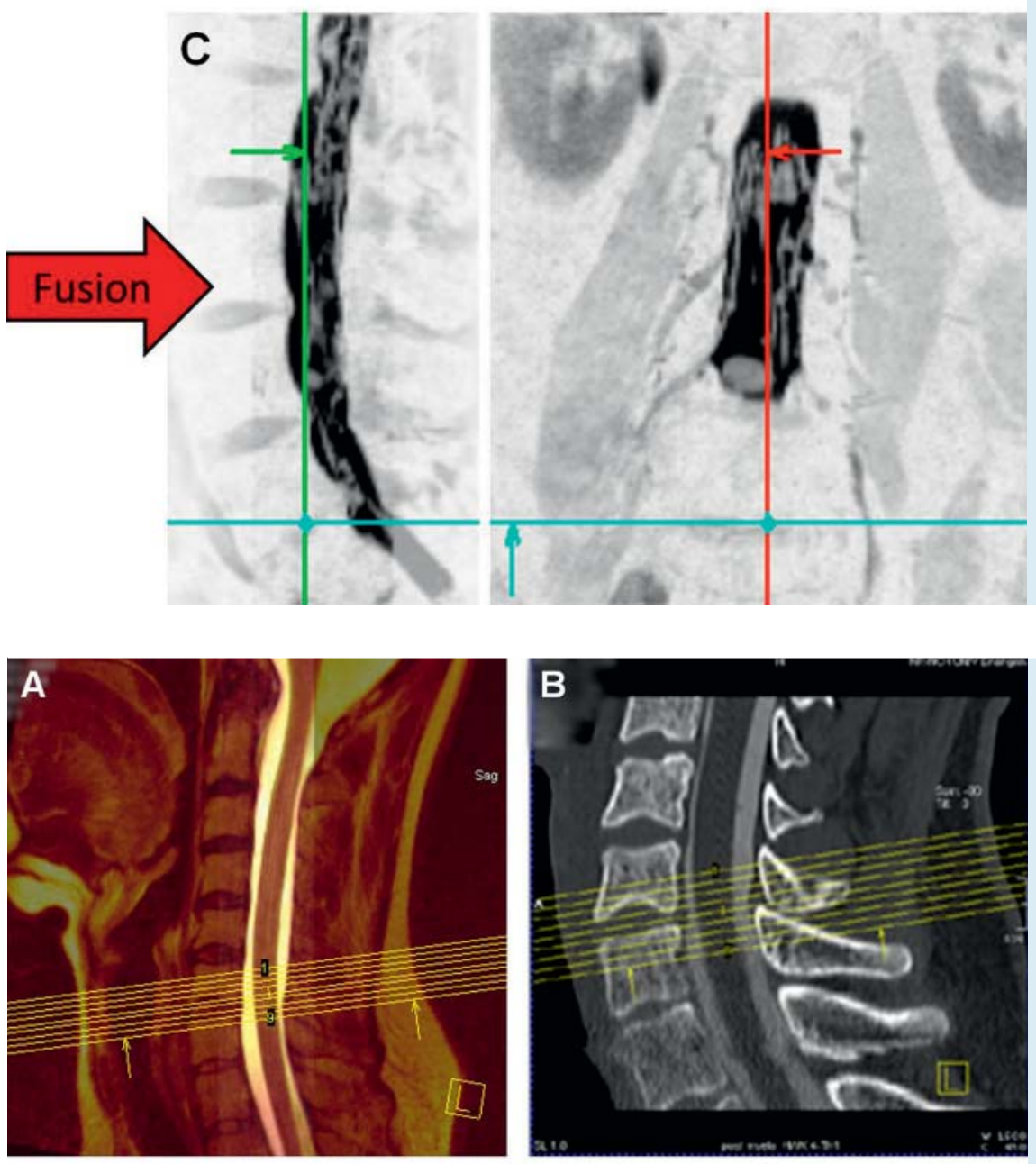

Abb. 3 Verbesserte MR-Myelografie und postmyelografisches $\mathrm{CT}$ bei einem Patienten auf der Höhe C6 / C7. Das Übersichtsbild a wurde mithilfe der Bildfusion angefertigt. b Übersichtsbild des postmyelografischen $C T$. Vergleich axialer Schnittbilder einer 3D-HASTE $\mathbf{c}$ mit denen eines postmyelografischen CT $\mathbf{d}$. Bei beiden Techniken sind die Nervenwurzeln klar als Aussparungen zu erkennen (rote Pfeile).
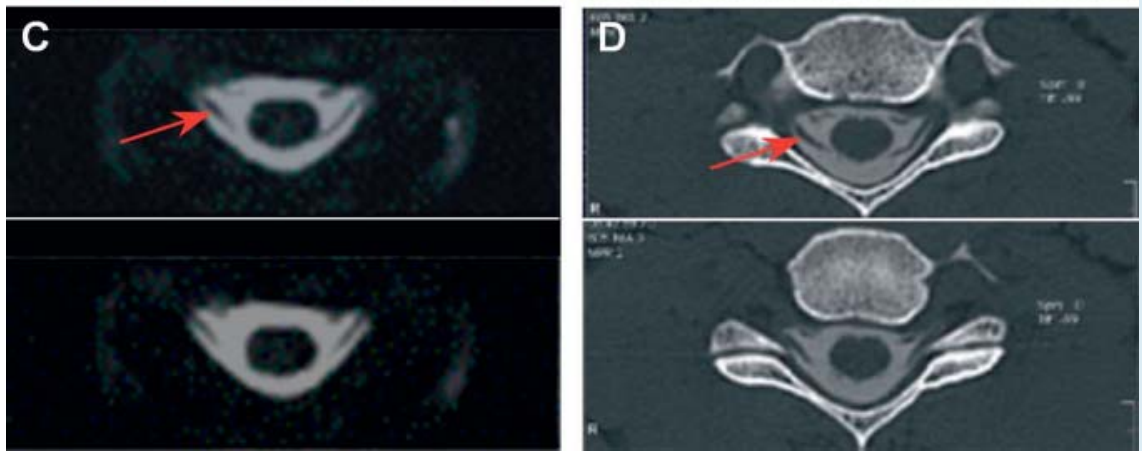

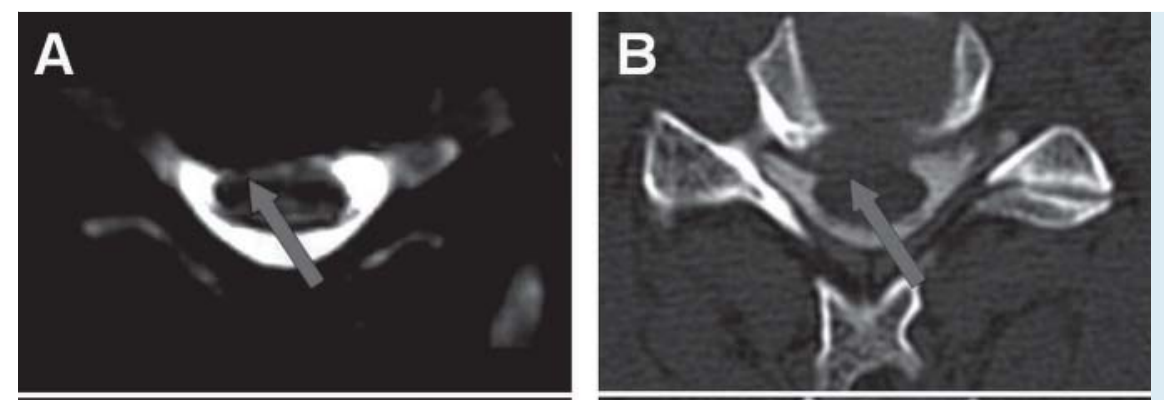

Abb. 4 Verbesserte MR-Myelografie und postmyelografisches $\mathrm{CT}$ bei einem Patienten mit Bandscheibenvorfall im Segment HWK 5/6. Axiale Schnittbilder der MR-Myelografie a und des postmyelografischen CT b des Zwischenwirbelraums C 5/6. Deutlich ist die Einengung des Liquorraums bei beiden Techniken zu sehen (graue Pfeile).
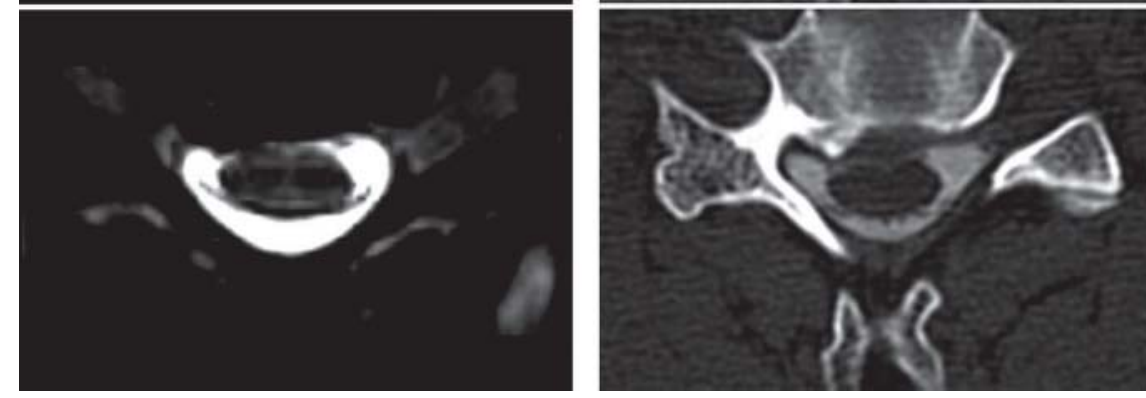

quenztechnik zu. Bei den sehr stark T2-gewichteten Liquorsequenzen kamen in der Vergangenheit oft Gradientenechosequenzen zum Einsatz [11, 26]. Nachteil dieser Sequenzen sind vor allem verstärkt auftretende Suszeptibilitätsartefakte in Regionen mit Strukturen unterschiedlicher Protonendichte [27]. In der Literatur ist der Einsatz von Spin-Echo-Sequenzen beschrieben [28, 29], deren Vorteil neben reduzierten Suszeptibilitätsartefakten auch das oft höhere Signal-zu-Rausch-Verhältnis ist [27].

In der vorliegenden Studie wurde eine 3D-Turbo-Spin-Echo-Sequenz (3D-HASTE) mit 4/8-Fouriertransformation und starker T2-Gewichtung verwendet. Um Signalüberlagerung durch Fettgewebe zu verhindern, wurde die Sequenz mit einer frequenzselektiven Fettunterdrückung verwendet. Die 4/8-Fouriertransformation („partial Fourier“) diente zur Unterdrückung der den Liquor umgebenden Strukturen. Hierbei bleiben die signalstarken liquorenthaltenden Strukturen unverändert gut abgrenzbar, während die Kontrastauflösung zur Umgebung hin maximal abnimmt, die umgebenden Strukturen erscheinen wie ausgelöscht. Ursache dieses Effekts ist die unterschiedliche Repräsentation grober und feiner Strukturen im Datenraum (k-Raum). Zur Verringerung der atmungsabhängigen Artefakte im Bereich der LWS sowie der Schluck- und Pulsationsartefakte im Bereich der HWS war die Sequenz außerdem mit zusätzlichen Sättigungspulsen ausgestattet.

Es zeigte sich, dass die signalarmen, intraduralen Substrukturen deutlich vom signalreichen Liquorraum abgrenzbar waren. Bei allen Patienten konnten die Spinalnerven der untersuchten Segmente eindeutig identifiziert werden. Bedingt durch die starke T2-Gewichtung und die Unterdrückung des Umgebungssignals geht allerdings die anatomische Zuordnung verloren. Somit kann die Segmentzugehörigkeit ungenügend überprüft werden, womit die Rekonstruktionsbilder für die Diagnose und die OPPlanung nicht mehr geeignet sind. Dieser entscheidende Nachteil wurde durch Bilddatenfusion mit einem anatomischen Datensatz hinreichend ausgeglichen.

Die in dieser Studie verwendete Strategie der Bildfusion von Datensätzen gleicher Modalität besitzt eindeutige Vorteile. Die Aufnahmen erfolgen im selben Untersuchungsgang und mit einer vergleichbaren Auflösung. Für eine rasche und exakte Fusion konnten die erforderlichen Kriterien definiert werden: Gleiche anatomisch Bezugspunkte müssen visualisiert sein und vergleichbare FoV. Der Grund liegt darin, dass der Fusionsalgorithmus charakteristische Anhaltspunkte in beiden Bildern benötigt. Diese können zum einen durch identische geometrische Strukturen (z. B. der Dens Axis im Bereich der HWS) oder gleiches Signalverhalten (z. B. gleiche Gewichtung des Liquorraums) gegeben sein. 3D-Datensätze können dann problemlos miteinander fusioniert werden, wenn ein gleiches FoV verwendet wurde, da hier in allen 3 Ebenen gut aufgelöste sekundäre Rekonstruktionen möglich sind. Nach Bildfusion ließen sich axiale Schnittbilder in definierten Höhen erstellen, die zusammen mit einem die Anatomie wiedergebenden Bild abgespeichert wurden. Die vergleichende Evaluierung des Fusionsergebnisses anhand des Goldstandards (postmyelografisches CT) ergab, dass alle untersuchten Segmente sowohl mit als auch ohne pathologischen Befund vergleichbare Ergebnisse in der Visualisierung zeigten. Vergleichbare Studien zur klinischen Bedeutung der Bildfusion unterschiedlicher MRDatensätze fehlen in der Literatur bisher.

Eine Schwäche der MR-Myelografie, so wie sie in dieser Studie durchgeführt wurde, ist sicher, dass Pathologien nur „statisch“ untersucht werden. Insbesondere die Detektion von Foramenstenosen im Stehen oder einer Zunahme der Bandscheibenprotrusion bzw. einer Spinalkanalstenose bei Reklination oder Inklination ist eine der wenigen noch verbliebenen Indikationen für eine konventionelle Myelografie in der heutigen Zeit. Um diese verbliebene Domäne der konventionellen Myelografie auch für die MR-Myelografie zu erschließen, wurden und werden Apparaturen entwickelt [30 - 34], um sogenannte Funktionsaufnahmen zu ermöglichen.

Die in dieser Arbeit verwendete Technik liefert dem aktuellen Goldstandard (postmyelografisches $\mathrm{CT}$ ) vergleichbare Visualisierungsergebnisse ohne den Einsatz von ionisierender Strahlung oder Kontrastmittel. Des Weiteren sind die Möglichkeiten durch die Software noch nicht vollständig ausgeschöpft. So ist es z. B. möglich, eine segmentbezogene Volumetrie des Liquorraums durchzuführen, was gerade in einer weiteren Studie untersucht wird. 


\section{Literatur}

1 Morishita Y, Hida S, Naito $M$ et al. Neurogenic intermittent claudication in lumbar spinal canal stenosis: the clinical relationship between the local pressure of the intervertebral foramen and the clinical findings in lumbar spinal canal stenosis. J Spinal Disord Tech 2009; 22: 130 134

2 Singh $K$, Samartzis D, Vaccaro AR et al. Congenital lumbar spinal stenosis: a prospective, control-matched, cohort radiographic analysis. Spine J 2005; 5: 615-622

3 Mayhew PD, Kapatkin AS, Wortman JA et al. Association of cauda equina compression on magnetic resonance images and clinical signs in dogs with degenerative lumbosacral stenosis. J Am Anim Hosp Assoc 2002; 38: 555-562

4 Schafer M, Riebeling H, Hacker $H$. False negative myelography in surgically confirmed lumbar intervertebral disk prolapse. Neurochirurgia 1976; 19: $201-207$

5 Hackenbroch MH, Waldecker B, Promper KH. Prolapse of the lumbar vertebral disk - correlation of $\mathrm{CT}$ and myelographic findings with surgical findings. Röntgenblätter 1983; 36: 50-55

6 Katayama H, Yamaguchi K, Kozuka $T$ et al. Adverse reactions to ionic and nonionic contrast media. A report from the Japanese Committee on the Safety of Contrast Media. Radiology 1990; 175: 621-628

7 Hausegger KA, Furstner M, Hauser M et al. Clinical application of flat-panel CT in the angio suite. Fortschr Röntgenstr 2011; 183: 1116-1122

8 Raininko $R$. The value of CT after total block on myelography. Experience with 25 patients. Fortschr Röntgenstr 1983; 138: 61 -65

9 Kampmann H, Schroedl P, Spranger M. Diagnosis of lumbar intervertebral disk prolapse by computed tomography. A comparative clinical study between myelographic, computed tomographic and the surgical research results with 158 patients. Röntgenblätter 1985; 38: 387-391

10 Janssen ME, Bertrand SL, Joe $C$ et al. Lumbar herniated disk disease: comparison of MRI, myelography, and post-myelographic CT scan with surgical findings. Orthopedics 1994; 17: 121-127

11 Sugahara T, Korogi Y, Hirai $T$ et al. Contrast-enhanced T1-weighted three-dimensional gradient-echo MR imaging of the whole spine for intradural tumor dissemination. Am J Neuroradiol 1998; 19: 1773-1779

12 Klasen J, Antoch G, Blondin D. MR imaging of the abdomen in pregnancy. Fortschr Röntgenstr 2011; 183: 514-522

13 Schaefer JF, Kramer U. Whole-body MRI in children and juveniles. Fortschr Röntgenstr 2011; 183: 24-36

14 Lemburg SP, Roggenland D, Nicolas $V$ et al. Analysis of image acquisition, post-processing and documentation in adolescents with spine injuries - comparison before and after referral to a university hospital. Fortschr Röntgenstr 2012; 184: 810-819

15 Freund M, Hutzelmann A, Steffens JC et al. MR myelography in spinal canal stenosis. Fortschr Röntgenstr 1997; 167: 474-478

16 Kuroki $\mathrm{H}$, Tajima $\mathrm{N}$, Hirakawa $\mathrm{S}$ et al. Comparative study of MR myelography and conventional myelography in the diagnosis of lumbar spinal diseases. J Spinal Disord 1998; 11: 487-492

17 Eberhardt KE, Hollenbach HP, Huk WJ. 3D-MR myelography in diagnosis of lumbar spinal nerve root compression syndromes. Comparative study with conventional myelography. Aktuelle Radiol 1994; 4: 313 317

18 Eberhardt KE, Hollenbach HP, Tomandl B et al. Three-dimensional MR myelography of the lumbar spine: comparative case study to X-ray myelography. Eur Radiol 1997; 7: 737-742

19 Ferrer P, Marti-Bonmati L, Molla E et al. Value of MR myelography in the diagnosis of the spine disorders. Med Clin 2000; 115: 366-369

20 Bartlett RJ, Hill CA, Devlin R et al. Two-dimensional MRI at 1.5 and $0.5 \mathrm{~T}$ versus $C T$ myelography in the diagnosis of cervical radiculopathy. Neuroradiology 1996; 38: $142-147$

21 Birchall D, Connelly D, Walker L et al. Evaluation of magnetic resonance myelography in the investigation of cervical spondylotic radiculopathy. Br J Radiol 2003; 76: 525-531

22 Melhem ER. Technical challenges in MR imaging of the cervical spine and cord. Magn Reson Imaging Clin N Am 2000; 8: 435-452

23 Wang YF, Lirng JF, Fuh JL et al. Heavily T2-weighted MR myelography vs CT myelography in spontaneous intracranial hypotension. Neurology 2009; 73: $1892-1898$

24 Bartynski WS, Lin L. Lumbar root compression in the lateral recess: MR imaging, conventional myelography, and CT myelography comparison with surgical confirmation. Am J Neuroradiol 2003; 24: 348 - 360

25 Borenstein DG, O'Mara JWJr, Boden SD et al. The value of magnetic resonance imaging of the lumbar spine to predict low-back pain in asymptomatic subjects: a seven-year follow-up study. J Bone Joint Surg Am 2001; 83-A: 1306 - 1311

26 Fenchel M, Roser F, Nagele $T$ et al. Syringomyelia - Syringomyelie. Fortschr Röntgenstr 2012; 184: 191 - 195

27 Maldjian C, Adam RJ, Akhtar $N$ et al. Volume fast spin-echo imaging of the cervical spine. Acad Radiol 1999; 6: 84-88

28 Maldjian C, Adam RJ, Akhtar $N$ et al. Volume (three-dimensional) fast spin-echo imaging of the lumbar spine. Acad Radiol 1999; 6: 339-342

29 Chiavassa H, Sans N, Galy-Fourcade D et al. HASTE sequence and cineMRI evaluation of the cervical spinal canal: evaluation in 11 healthy subjects. J Radiol 2000; 81: 611-617

30 Kanno H, Ozawa H, Koizumi Y et al. Dynamic change of dural sac crosssectional area in axial loaded magnetic resonance imaging correlates with the severity of clinical symptoms in patients with lumbar spinal canal stenosis. Spine (Phila Pa 1976) 2012; 37: 207-213

31 Kanno $\mathrm{H}$, Endo T, Ozawa $\mathrm{H}$ et al. Axial loading during magnetic resonance imaging in patients with lumbar spinal canal stenosis: does it reproduce the positional change of the dural sac detected by upright myelography? Spine (Phila Pa 1976) 2012; 37: E985 - E992

32 Allmann $K H$, Schafer $O$, Uhl M et al. Kinematic versus static MRI study of the cervical spine in patients with rheumatoid arthritis. Fortschr Röntgenstr 1999; 170: $22-27$

33 Muhle C, Brossmann J, Biederer J et al. Value of kinematic MRI in the evaluation of patients with exacerbated pain in cervical spine motion compared with static MRI. Fortschr Röntgenstr 2001; 173: 126-132

34 Schlamann M, Reischke L, Klassen D et al. Dynamic magnetic resonance imaging of the cervical spine using the NeuroSwing System. Spine (Phila Pa 1976) 2007; 32: $2398-2401$ 